\title{
Passive sampling of organic contaminants across the water-sediment interface of an urban stream
}

Jonas Mechelke ${ }^{1,2}$, Etiënne L.M. Vermeirssen ${ }^{3}$, Juliane Hollender ${ }^{1,2^{*}}$

${ }^{1}$ Eawag, Swiss Federal Institute of Aquatic Science and Technology, 8600 Dübendorf, Switzerland

${ }^{2}$ Institute of Biogeochemistry and Pollutant Dynamics, ETH Zurich, 8092 Zürich, Switzerland

${ }^{3}$ Swiss Centre for Applied Ecotoxicology, 8600 Dübendorf, Switzerland

* Corresponding Author: Juliane Hollender, ORCID: 0000-0002-4660-274X, juliane.hollender@eawag.ch, +41 587655493

\section{Graphical abstract}
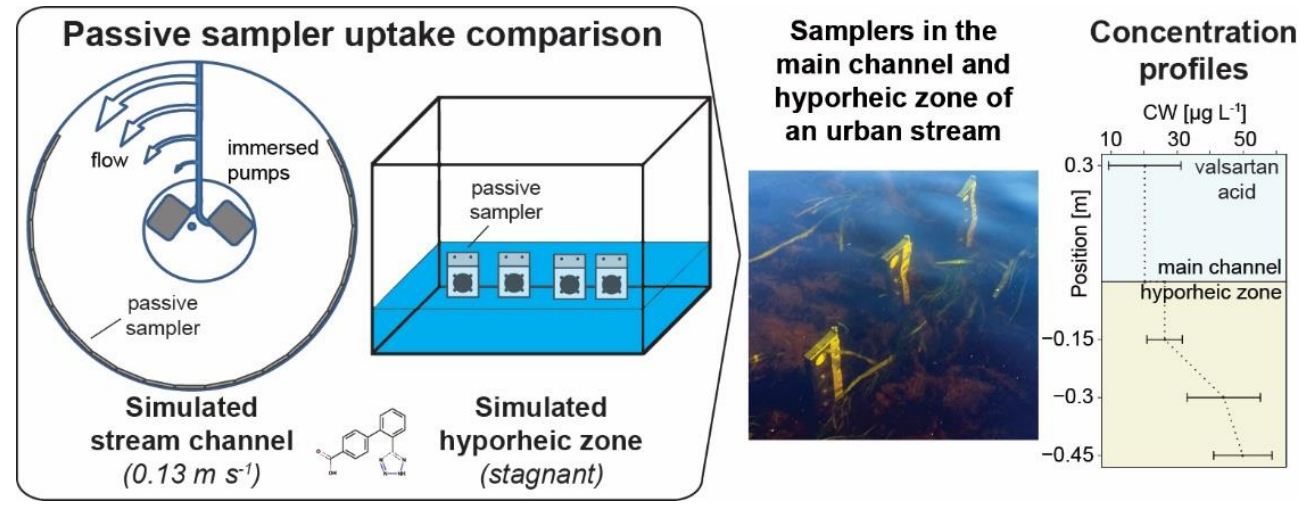

This document is the accepted manuscript version of the following article:

Mechelke, J., Vermeirssen, E.L.M., Hollender, J., 2019. Passive sampling of organic contaminants across the water-sediment interface of an urban stream. Water Research. https://doi.org/10.1016/ j.watres.2019.114966

This manuscript version is made available under the CC-BY-NC-ND 4.0 license http:// creativecommons.org/licenses/by-nc-nd/4.0/ 


\section{Abstract}

Passive sampling is a well-established tool for monitoring time-weighted average concentrations of polar and semipolar organic contaminants in streams at flow velocities between 0.1 and $0.4 \mathrm{~m} \mathrm{~s}^{-1}$. However, its application under low-flow conditions $\left(10^{-5}\right.$ to $\left.0.01 \mathrm{~m} \mathrm{~s}^{-1}\right)$ - as encountered in hyporheic zones - has been scarcely reported. In this study, 3 novel passive sampler configurations were developed for the monitoring of (semi-)polar organic pollutants and related transformation products across the water-sediment interface and thus across varying hydrodynamic conditions. Their design was inspired by Chemcatcher and diffusive gradients in thin films for organics. To determine the most optimal sampler design, an uptake experiment was completed involving the 3 novel passive sampler configurations and a reference Chemcatcher in polar configuration. The experiments consisted of a circular flume that simulated the main channel of a stream and an aquarium with stagnant water that represented the underlying hyporheic zone. The systems were exposed to 192 organic pollutants at environmental concentrations, and the samplers were then collected, extracted and analyzed using liquid chromatography high-resolution mass spectrometry after 2, 6 and 14 days. The configuration that was most insensitive to different hydrodynamic conditions consisted of a reversed-phase sulfonated styrenedivinylbenzene disk as the receiving phase that was covered by an agarose diffusion gel and topped with a polyethersulfone membrane filter. To further evaluate its environmental application, samplers were installed downstream of a sewage treatment plant located at an urban

stream in Berlin, Germany (Erpe). The samplers were mounted on custom-made holders which were subsequently embedded in the stream bed to position samplers above $(0.30 \mathrm{~m})$ and within the sediment $(-0.15 /-0.30 /-0.45 \mathrm{~m})$ for 11 days. Target and suspect screening workflows were then applied to identify common concentration patterns and link parent attenuation to transformation product formation. A total of 104 concentration profiles were determined, suggesting the efficiency of the proposed sampling strategy in the water-sediment interface. Valsartan acid was the only known transformation product indicative of hyporheic zone-driven attenuation as its concentration in porewater by far exceeded its concentration in surface water. Similar patterns were observed for a larger list of suspected transformation products, of which a sotalol transformation product was tentatively identified. Overall, the established sampling methodology can be effectively used to quantify organic contaminants during low-flow conditions and is suitable for the characterization of attenuation patterns of organic pollutants in hyporheic zones.

\section{Keywords}

o-DGT, Chemcatcher, micropollutants, hyporheic zone, urban stream, transformation products 


\section{Introduction}

Many synthetic organic chemicals (OCs) enter the urban water cycle via sewage systems and end up in surface waters via discharge from sewage treatment plants (STPs) (Schwarzenbach et al., 2006). Upon release, they can undergo several abiotic and biotic processes such as dilution, photolysis, hydrolysis, sorption and biotransformation (Schwarzenbach et al., 2016). One possible but rarely studied process is the attenuation in hyporheic zones (HZs). $\mathrm{HZs}$ are water-saturated subsurface environments in lotic systems that emerge when surface water (SW) downwells into subsurface flow paths. Water in the $\mathrm{HZ}$ may travel for some distance beneath the stream bed or inside stream banks (millimeters to kilometers in seconds to months), possibly mix with groundwater and finally return to the active channel (Malard et al., 2002). During this residence, OCs can encounter excellent conditions for biotransformation due to microbially diverse biofilms, steep physico-chemical gradients, constant input of electron acceptors and donors with infiltrating SW and an abundance of sorption sites. Owing to these characteristics, the $\mathrm{HZ}$ has been hypothesized to act as natural fixed-bed bioreactor and a central compartment for pollutant attenuation. It thus contributes to the self-purification of lotic systems and serves as a barrier against nearsurface aquifer contamination (Lewandowski et al., 2011b). To date, only few studies have tested these hypotheses. Studies with explicit focus on the fate of OCs in HZs were either conducted in bench-scale flumes (Kunkel and Radke, 2008; Li et al., 2015), in a sediment core of a river bank filtration site (Burke et al., 2014) or insitu river field sites (Jaeger et al., 2019; Kunkel and Radke, 2012, 2011; Lewandowski et al., 2011b; Posselt et al., 2018; Radke et al., 2010; Schaper et al., 2019, 2018b). In these studies, HZ-like conditions have shown to enhance or induce the biotransformation of certain OCs. Hence, some biological transformation products (TPs) were exclusively formed in the $\mathrm{HZ}$, transported back to SW and therefore proposed as potential indicators of hyporheic transformation (Li et al., 2015). In one of the most recent lab studies on the hyporheic fate of polar OCs Li et al. (2015) concluded that carefully designing field experiments is important to determine the exact contribution of $\mathrm{HZ}$ transformation of OCs in SWs.

Polar ( $\mathrm{pH}$ dependent octanol-water distribution coefficient, i.e. $\left.\log \mathrm{D}_{\mathrm{ow}} \leq 1\right)$ and semi-polar $\left(1<\log \mathrm{D}_{\mathrm{ow}} \leq 3\right.$, apolar: $\log \mathrm{D}_{\text {ow }}>3$ ) neutral or anionic OCs are especially suitable for studying hyporheic transformation via biodegradation. They do not or only slightly sorb to predominantly negatively charged natural surfaces (in particular sediment organic matter) and thus possess a high subsurface mobility. The latter suggests that biodegradation is the highest contributor to their overall loss. Cationic or non-polar neutral compounds on the other hand tend to sorb and are less mobile (Reemtsma et al., 2016). Furthermore, tracing OCs and their biological TPs in field experiments in the $\mathrm{HZ}$ over an extended time-period requires sampling techniques that preserve sediment integrity and do not (or only 
mildly) alter the hyporheic flow paths. This rules out ex-situ porewater (PW) sampling approaches, where sediment is collected and PW extracted offsite (Gruzalski et al., 2016). Hence, in-situ sampling is the preferred method, provided it is minimally invasive. Examples of active in-situ sampling approaches are the USGS minipoint sampler (Duff et al., 2007), modified versions thereof (Posselt et al., 2018; Schaper et al., 2018a) and the USEPA drive point sampler (USEPA, 2013). Passive in-situ devices include peeper dialysis samplers (Teasdale et al., 1995), gel samplers (Krom et al., 1994) and diffusive gradients in thin films (DGT) for nutrients and trace metals (Davison and Zhang, 1994).

A passive sampler (PS) is a device that is deployed over an extended time period (days to weeks) in a medium (e.g. SW, sediment) where it accumulates the analytes of interest. If designed properly, it can operate in timeintegrative mode such that a time-weighted average (TWA) concentration of a target analyte can be inferred from its accumulated mass (Yongping Zeng et al., 2011). While this comes with a loss of temporal resolution, an advantage of passive sampling is its minimal supervision over the sampling period. By contrast, active sampling approaches require frequent monitoring but can be automated to capture multiple snapshots over time. PSs are typically used in streams at flow velocities between approximately 0.1 and $0.4 \mathrm{~m} \mathrm{~s}^{-1}$. Applications under low-flow conditions $\left(10^{-5}\right.$ to $\left.0.01 \mathrm{~m} \mathrm{~s}^{-1}\right)$ as encountered in groundwater or HZs (Boulton et al., 2002) have been scarcely reported. Examples are the uptake of antibiotics into o-DGT at different stirring speeds (including 0 rpm) (Chen et al., 2012) and in wetted soils (Chen et al., 2014). When discussed in the literature, low-flow PS applications typically target hydrophobic OCs using polydimethylsiloxane or polyethylene receiving phases operated in equilibrium mode (Lydy et al., 2014). The time-integrative passive sampling of hydrophobic OCs in sediment PW (Liu et al., 2013) is less common, and its application on polar OCs in $\mathrm{HZs}$ is virtually unexplored.

Passive sampling across the water-sediment interface is not straightforward. Flow velocities in SW and $\mathrm{HZ}$ can easily differ by several orders of magnitude (see above) and since flow velocity is a major driver for uptake into PSs (Vermeirssen et al., 2008), OC uptake in the 2 compartments will often differ due to this flow velocity difference. A quantitative comparison of SW and $\mathrm{HZ}$ chemistry via PSs cannot be performed and will only be meaningful after PS calibration at flow velocities encountered in both compartments. An alternative is the use of PSs that are insensitive to hydrodynamics, especially since low-flow calibration at velocities in the $\mathrm{m} \mathrm{d}^{-1}$ range $\left(10^{-5} \mathrm{~m} \mathrm{~s}^{-1}\right)$ is difficult to establish and measure experimentally. PSs virtually unaffected by hydrodynamics due to thick uptake limiting barriers are ceramic dosimeters (Bopp et al., 2005) and DGT for nutrients and trace metals (Davison and Zhang, 1994). More recent developments are DGT for organics (o-DGT) (Challis et al., 2016; Chen et al., 2014, 2013, 2012, 2018, 2017; Guan et al., 2018; Guibal et al., 2017; Guo et al., 2017; Xie et al., 2018a, 2018b; Zhang 
et al., 1998) and a ceramic dosimeter for polar organics (Cristale et al., 2013). Although the use of such PSs might eliminate the need for calibration, uptake by these PSs is significantly reduced. Low uptake, however, limits a robust quantification of OCs or requires impractically long deployment periods (up to 150 days for polycyclic aromatic hydrocarbons in ceramic dosimeters (Bopp et al., 2005)). The ideal device for passive sampling across the watersediment interface would therefore (i) feature uptake limiting barriers that render uptake insensitive to changes in hydrodynamic conditions, (ii) accumulate sufficient analyte required for instrumental analysis and (iii) respond to fluctuating environmental concentrations within the desired deployment period.

The primary aim of this study was to develop such PS that can be applied for the monitoring of OCs and related TPs across the water-sediment interface within the time frame of days. Using a PS that is less sensitive to hydrodynamics than commonly employed PSs was the major attempt to minimize uncertainty in its field applications. Thus, we designed 3 types of passive sampler configurations which are based on the features of Chemcatcher and o-DGT. Chemcatcher is the most widely used PS for monitoring polar OCs in SW together with the Polar Organic Chemical Integrative Sampler (Booij and Chen, 2018). For polar application, Chemcatchers are typically equipped with disks made of reversed-phase sulfonated styrenedivinylbenzene (SDB-RPS) copolymeric beads embedded in polytetrafluoroethylene (10 wt.\%) as the receiving phase. By contrast, o-DGT feature a binding gel as the receiving phase topped with an agarose diffusion gel. The binding gel (also made from agarose) contains sorbent material selected according to the desired affinity towards target analytes, such as hydrophilic lipophilic balanced (HLB) copolymer for the enrichment of semi-polar to non-polar substances. Both Chemcatcher and oDGT can be used with or without polyethersulfone (PES) membrane that limits uptake and serves as a mechanical protection. After PS design and the evaluation of uptake, 1 of the 3 novel PS configurations was selected and employed in the field to gain insights on the occurrence and HZ-driven transformation of a wide variety of organic micropollutants.

\section{Materials and Methods}

\subsection{Chemicals, solutions and calibration series}

Reference standards (STDs) and isotope-labeled internal standards (ISs) were purchased from CDN Isotopes (Canada), Dr. Ehrenstorfer (Germany), HPC Standards (Germany), LGC Standards (Switzerland), Molcan (Canada), MolPort (Latvia), Monsanto (Belgium), Novartis (Switzerland), Riedel-de-Häen (Germany), SigmaAldrich (Switzerland) or Toronto Research Chemicals (Canada) at purities $\geq 95 \%$ (analytical grade). NANOpure ${ }^{\mathrm{TM}}$ 
water (NPW) was generated with a lab water purification system (D11911, Barnstead/Thermo Scientific, USA). Acetone, ethanol and methanol were of LC/MS grade (Optima ${ }^{\mathrm{TM}}$, Fisher Scientific, Switzerland), formic acid ( $\geq 98 \%$, Merck, Germany) and agarose (white powder form, low EEO, Promega, Switzerland) of analytical grade. STD and IS stock solutions (1'000 or $100 \mathrm{mg} \mathrm{L}^{-1}$ ) were prepared in suitable solvents and combined as mixtures. STD mixtures were then combined as a stock solution $\left(2.5 \mathrm{mg} \mathrm{L}^{-1}\right)$ from which 2 calibration series were prepared, i.e. the first in methanol for the quantification of PS extracts $\left(0,1,5,10,50,100,500\right.$ and 1 ' $\left.000 \mu \mathrm{L} \mathrm{L}^{-1}\right)$, and the second in NPW $\left(0,5,10,100,250,500,750,1^{\prime} 000\right.$ and $\left.10^{\prime} 000 \mathrm{ng} \mathrm{L}^{-1}\right)$ to quantify water samples. The remaining stock solution (2.5 $\left.\mathrm{mg} \mathrm{L}^{-1}\right)$ was then evaporatively concentrated to approx. $50 \mathrm{mg} \mathrm{L}^{-1}$ to be used as dosing/spiking solution. The evaporation step served to minimize the organic carbon content in dosing solutions (approx. $50 \mathrm{mg} \mathrm{L}^{-1}$ methanol) and finally to prevent bacterial growth in the exposure media. Aliquots of the concentrated stock solution were used to set the initial OC level of approx. $600 \mathrm{ng} \mathrm{L}^{-1}$ in the 2 PS uptake experiments and for the preparation of dosing solutions. OC levels in dosing solutions ranged from 3'700 to $10^{\prime} 000 \mathrm{ng} \mathrm{L}^{-1}$ (mean: 7'500 $\mathrm{ng} \mathrm{L}^{-1}$ ) and thus deviated from the target concentration of $8^{\prime} 400 \mathrm{ng} \mathrm{L}^{-1}(0.42 \mathrm{~mL}$ concentrated stock solution in $2.5 \mathrm{~L} \mathrm{NPW})$ indicating possible OC loss during evaporation or in the subsequent preparation of the dosing solution. An exhaustive OC list can be found in the Electronic Supplementary Material (ESM, Table ESM2-1).

\subsection{Design, preparation and assembly of passive sampler configurations}

Three novel PS configurations (PS1 to PS3 in Fig. 1) were designed by combining and modifying the components of Chemcatcher and o-DGT. Modifications were meant to increase solute transfer resistance in the uptake limiting barrier such that the uptake resistance was governed by this barrier and less by hydrodynamic conditions (i.e. by the water flow velocity and thus the resistance over the water boundary layer). A Chemcatcher in polar configuration served as reference PS (PS-R) and consisted of an Empore ${ }^{\mathrm{TM}}$ SDB-RPS disk (Model 2241, 3M, Switzerland, diameter: $47 \mathrm{~mm}$, thickness: $0.5 \pm 0.05 \mathrm{~mm}$ ) as the receiving phase topped with a PES membrane (Supor 100, PALL, Switzerland, diameter; $47 \mathrm{~mm}$, pore size: $0.1 \mu \mathrm{m}$, thickness: 132 to $145 \mu \mathrm{m})$. PS1 was a PS-R with a second PES membrane (SDB-RPS disk/PES membrane/PES membrane). PS2 was an o-DGT topped with a PES membrane (binding gel/diffusion gel/PES membrane). It was inspired by o-DGT described by Chen et al. (2012), but featured a 4 times larger exposed surface area $\left(12.6 \mathrm{~cm}^{2}\right.$ against $3.1 \mathrm{~cm}^{2}$ in the standard DGT housing) and a different receiving phase (SDB-RPS against XAD18). PS3 was a combination of PS1 and PS2, i.e. SDB-RPS disk was the receiving phase topped with a diffusion gel and covered by a PES membrane (SDB-RPS disk/diffusion gel/PES membrane). 
For binding gel preparation, $3.5 \mathrm{~g}$ sorbent material (Oasis HLB, $10 \mu \mathrm{m}$, Waters, Switzerland) were wetted in $20 \mathrm{~mL}$ NPW for $10 \mathrm{~min}$ under stirring. The slurry was centrifuged $\left(3^{\prime} 020 \mathrm{~g}, 5 \mathrm{~min}, 20^{\circ} \mathrm{C}\right)$ and the supernatant discarded. Six $\mathrm{g}$ of wet sorbent (of $7 \mathrm{~g}$ in total) were stirred in $15 \mathrm{~mL}$ hot NPW and combined with $15 \mathrm{~mL}$ hot agarose solution $(2 \% \mathrm{w} / \mathrm{v})(\mathrm{NPW}$ and agarose solution were both brought to the boil in a microwave and allowed to cool briefly before further use). This suspension ( $1 \% \mathrm{w} / \mathrm{v}$ agarose) was cast between Perspex plates of a custom-made casting device. The diffusion gel was prepared from $30 \mathrm{~mL}$ hot agarose solution $(1 \% \mathrm{w} / \mathrm{v})$ without the addition of sorbent material. Both gels were left to cool and solidify at $4^{\circ} \mathrm{C}$ inside the casting device. Finally, $47 \mathrm{~mm}$ disks were cut out with a punch-cutter and stored in NPW at $4^{\circ} \mathrm{C}$. SDB-RPS disks and PES membranes were conditioned in methanol (30 min) and subsequently in NPW (30 min) on a rotary shaker. The NPW was exchanged and both were stored at $4^{\circ} \mathrm{C}$ until PS assembly. All PSs were assembled in a housing that provided a 4 times larger exposed area than the standard DGT housing. For the assembly of PSs (Fig. 1), the receiving phase was placed on a stainless steel plate (70 by $100 \mathrm{~mm}$ ), eventually topped with a diffusion gel (PS2, PS3), covered by 1 (PS2, PS3, PS-R) or 2 (PS1) PES membranes and closed by a stainless steel cover plate $(70$ by $70 \mathrm{~mm})$. Polytetrafluoroethylene spacer plates were used to avoid excessive squeezing of gels while keeping the layers in place. Assembled PSs were stored in NPW at room temperature until use in the uptake experiment (section 2.4) or in the field (section 2.5). 


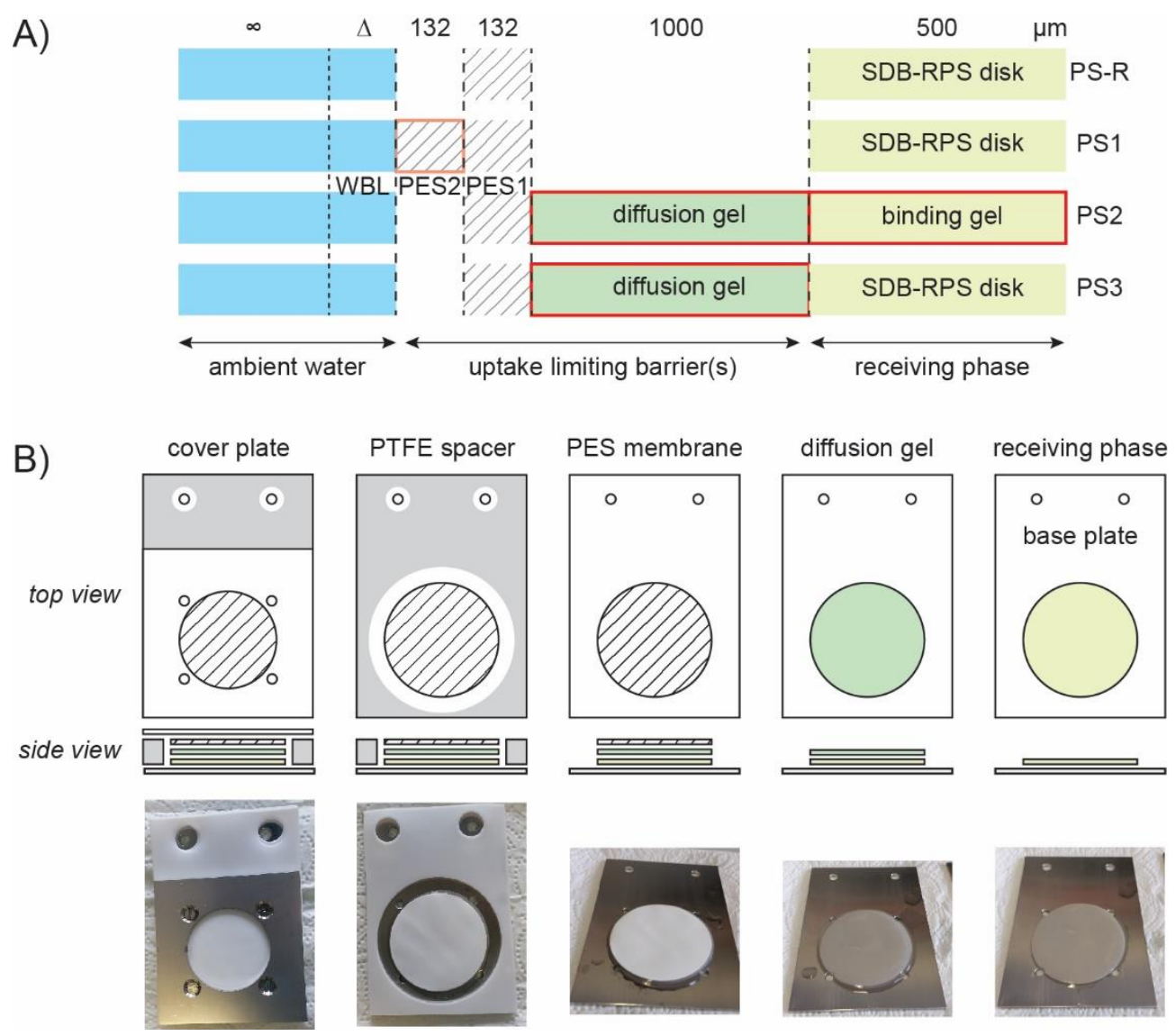

Fig. 1 Passive sampler (PS) design for the 3 novel configurations (PS1 to PS3) and a commonly used Chemcatcher in polar configuration as reference (PS-R). A: sampler cross sections. Modifications as compared to PS-R are highlighted in red. B: assembly sequence exemplarily shown for PS3 (from right to left). Abbreviations: PES: polyethersulfone membrane. PTFE: polytetrafluorethylene. WBL: water boundary layer.

\subsection{Field site selection and substance screening}

Within the HypoTRAIN project, a stretch of the urban stream Erpe in Berlin, Germany was selected as the field site for a joint experiment on the hyporheic fate of OCs. The Erpe is a eutrophic urban lowland stream at the eastern edge of Germany that receives up to $80 \%$ of its discharge as effluent from several smaller and one large municipal STP (Muenchehofe, 42'500 $\mathrm{m}^{3} \mathrm{~d}^{-1}$ dry weather capacity). The daily and weekly fluctuations in STP effluent discharge, effluent loads, and superimposed precipitation events typically result in considerable changes in SW composition (Lewandowski et al., 2011b; Posselt et al., 2018). The SW is therefore characterized by a high OC and nutrient load, resulting in a fine-sandy siliceous, predominantly clogged anoxic downstream sediment with a high organic carbon content (Gücker et al., 2006). Although the latter characteristics do not suggest a HZ, the large cross-sectional storage zone (Gücker and Pusch, 2006) potentially contributes to HZ formation. 
In September 2015, about a year prior to the actual joint field experiment in June 2016, Chemcatchers (PS-R) were deployed for 2 weeks (September 15-29) up- and downstream of STP Muenchehofe to screen for OCs and known TPs. Over 300 OCs were quantified (see Table ESM2-1), 60 of which were found in up- and downstream samples above the limit of quantification (LOQ). Additional 83 OCs were found only in downstream samples. Several of these OCs were selected to characterize uptake into the different PS configurations. Of particular interest were TPS and (semi-)polar neutral or anionic OCs for their suitability and applicability in hyporheic fate studies. The selection was further extended to cover a wide range of physico-chemical substance properties, such as polarity (log $\mathrm{D}_{\mathrm{ow}, \mathrm{pH}} \mathrm{B} .3$ -4.6 to 5.9), speciation (75 neutral, 45 anionic, 52 cationic, 20 zwitterionic) and molecular weight (102 to $821 \mathrm{Da}$ ). The $\log \mathrm{D}_{\text {ow }}$ values were predicted from SMILES strings using JChem for Excel (Vers. 18.8.0.253, ChemAxon), OC speciation at a particular pH using ChemAxon's Calculator (cxcalc) in batch mode. The final substance selection for the uptake experiment consisted of 192 OCs of various substance classes (see Table ESM2-1, 127 thereof were found in the Erpe).

\subsection{Passive sampler uptake experiments}

Uptake into PS1 to PS3 was evaluated and compared against PS-R under 2 hydrodynamic conditions: (i) in a circular flume (made of Perspex, partly described in (Vermeirssen et al., 2013)) at continuous flow simulating the hydrodynamics of a stream channel and (ii) in a glass aquarium with stagnant water (no forced flow) simulating hydrodynamic conditions similar to those encountered in $\mathrm{HZs}$ (a flow velocity close to zero or in the low $\mathrm{m} \mathrm{d}^{-1}$ range, a rough estimate of the convective flow velocities in the aquarium at nearly constant water temperature). Schemes and photographs of the 2 setups are shown in the ESM (Fig. ESM1.B-1, Fig. ESM1.D-2).

PSs were exposed in duplicates to the 192 selected OCs in tap water at environmentally relevant concentrations (approx. $600 \mathrm{ng} \mathrm{L}^{-1}$ ) and removed from the 2 basins after 2, 6 and 14 days. PS duplicates kept as lab blanks in NPW inside a field box near the experimental setup were collected after 14 days. After retrieval, the exposed part of SDB-RPS disks and binding gel were cut out (circular diameter: $40 \mathrm{~mm}$ ), SDB-RPS disks were carefully dried using paper towels, and both were stored in $6 \mathrm{~mL}$ acetone at $-20^{\circ} \mathrm{C}$ in $7.5 \mathrm{~mL}$ brown glass vials until extraction.

While more than half of the spiked circular flume water was renewed per day ( $20 \mathrm{~L}$ of in total $37.7 \mathrm{~L}$ ), the water in the aquarium was not renewed to minimize water movement and only received an initial OC spike. For water renewal, tap water was pumped from a reservoir into the flume using a rotary vane pump $\left(833 \mathrm{~mL} \mathrm{~h}^{-1}\right)$. At the end of the feeding tube, the dosing solution (section 2.1) was infused with a HPLC pump (60 $\left.\mathrm{mL} \mathrm{h}^{-1}\right)$. 
$\mathrm{OC}$ concentrations, water temperature and $\mathrm{pH}$ in the basins were monitored in daily water grab samples (stored at $-20^{\circ} \mathrm{C}$ until analysis). Both setups were covered with lightproof pond liner to reduce evaporation and exposure to ambient light. The water in the round basin was circulated using 2 immersion pumps (compact 1000, Eheim). Since the water flow velocity along the edge of the basin was not fully homogenous, PSs were shifted by 3 positions (24 positions in total) counter-clockwise every $24 \mathrm{~h}$ and were therefore exposed to approximately the same mean flow velocity of $0.13 \mathrm{~m} \mathrm{~s}^{-1}$ (Table ESM1.C-2).

If the mass of an OC was increasing over time in the receiving phase of a PS, the sampling rate (Rs) was calculated according to Eq. (1) for every time-point sampled ( $t=2,6$ and 14 days). The Rs is described in terms of volume of water extracted by a PS per unit time and is typically reported in liters or milliliters per day.

Eq. (1) $\mathrm{Rs}_{\mathrm{OC}}(\mathrm{t})_{\mathrm{t}=2,6 \text { or } 14 \text { days }}=\frac{\text { mean } \mathrm{m}_{\mathrm{OC}} \text { in receiving phase after time } \mathrm{t}}{\mathrm{OC} \text { concentration in water }}$

Rss obtained for 2, 6 and 14 days were averaged but only further considered if linearity of uptake was fulfilled (RSD among Rss $\leq 30 \%$ ). In case of a short lag-phase, i.e. the absence of an OC in PS extracts of day 2, only the Rss of days 6 and 14 were averaged. For a longer lag-phase ( $\geq 6$ days, i.e. absence of the OC in PS extracts of day 2 and 6) linearity could not be evaluated and Rs calculation and subsequent OC evaluation was not performed.

\subsection{Field application in the hyporheic zone of an urban stream}

OCs were passive sampled across the water-sediment interface of urban stream Erpe downstream of STP Muenchehofe (52 $\left.28^{\prime} 44.4^{\prime \prime} \mathrm{N} 13^{\circ} 38^{\prime} 08.6^{\prime \prime} \mathrm{E}\right)$ from June 5-16, 2016 using the most effective PS (PS3). The latter was identified by the results of the uptake experiments applying the following criteria: insensitivity towards changing hydrodynamic conditions and physical robustness in the field. Assembled PS3 were stored in NPW inside a field box, transported to the field and mounted on custom-made holding devices. Three holders were embedded in the sediment, positioning the PSs in SW $(0.30 \mathrm{~m}$ above the sediment) and within the sediment along (theoretical) horizontal (0.3 m distance, holder 1, 2 and 3 ) and vertical (-0.15/-0.30/-0.45 m) transects (Fig. 2). To account for the unwanted OC contamination, additional PSs (PS-R) were transported within the field box but were not deployed in the stream (i.e. stored inside the field box over the deployment period as field blanks). After 11 days, PSs (stream and field box) were retrieved, carefully dried using paper towels, receiving phases cut out (40 mm diameter), stored at $-20^{\circ} \mathrm{C}$ and transported to Eawag Dübendorf, Switzerland on dry ice. Upon arrival, receiving phases were immersed in $6 \mathrm{~mL}$ acetone and stored at $-20^{\circ} \mathrm{C}$ until extraction. 


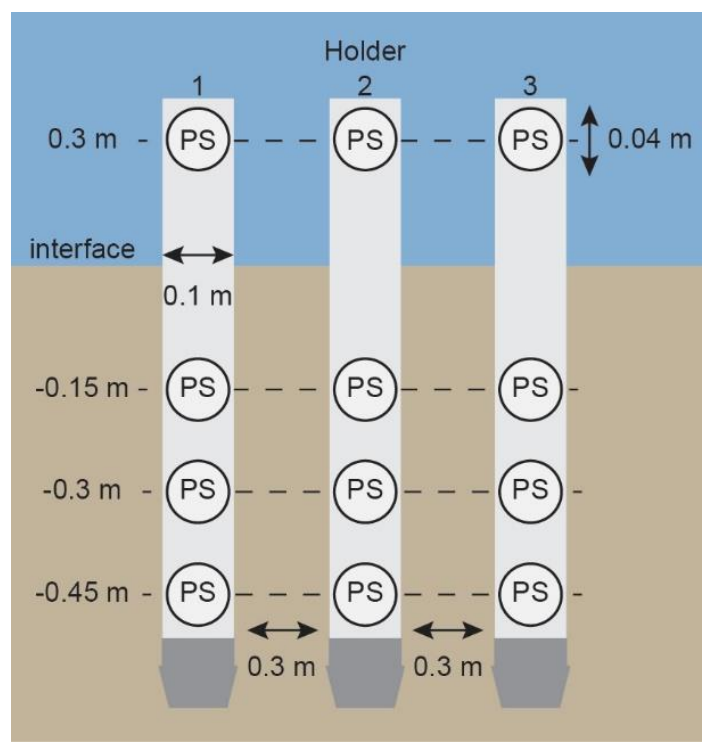

Fig. 2 Schematic illustration of the passive sampler (PS) installation in the main channel and porewater of the urban stream Erpe along theoretical horizontal and vertical transects.

Holder dimensions: $0.84 \times 0.10 \times 0.01 \mathrm{~m}(\mathrm{~L} \times \mathrm{W} \times \mathrm{H})$.

Flow velocity in SW was measured immediately next to the PSs directly after installation (June 5, 10 a.m., $0.10 \mathrm{~m}$ $\mathrm{s}^{-1}$ ) and after 6 days of deployment (June 11, 8 a.m., $0.08 \mathrm{~m} \mathrm{~s}^{-1}$ ) using an electromagnetic current meter with velocity and depth sensor (OTT MF Pro). PSs were inspected daily for the accumulation of macrophyte debris and driftwood, and cleaned if necessary. The saturated hydraulic conductivity was determined (KSAT, METER, Germany) in a sediment core extracted directly next to the PS holders. In the top sediment layer (0 to $0.05 \mathrm{~m})$ hydraulic conductivity was $8.1 \times 10^{-5} \mathrm{~m} \mathrm{~s}^{-1}\left(7 \mathrm{~m} \mathrm{~d}^{-1}, 25.3^{\circ} \mathrm{C}\right)$ and was slightly lower in the adjacent layer $(0.07$ to $0.12 \mathrm{~m})$ at $6.4 \times 10^{-5} \mathrm{~m} \mathrm{~s}^{-1}\left(5.5 \mathrm{~m} \mathrm{~d}^{-1}\right)$ (personal communication: Anne Mehrtens). In another sediment core, free dissolved oxygen was measured across the water-sediment interface directly in the field using an oxygen probe, revealing that PW was anoxic below $0.005 \mathrm{~m}$ (personal communication: Malte Posselt). In a parallel study conducted in nearby sediment (Schaper et al., 2019), free dissolved oxygen concentrations were in the same range (personal communication: Malte Posselt), however, further interpretation of nutrient species (nitrate, iron, manganese, dissolved organic carbon) led to the conclusion that oxic conditions must have prevailed in the upper $0.30 \mathrm{~m}$ with suboxic conditions below. Sediment organic carbon fractions reported in the literature for nearby sediment patches are elevated and of high spatial variability with 0.5 to 6 wt.\% (55 m downstream of the PS field site) (Schaper et al., 2018b) and 8.7 wt.\% (100 m downstream) (Raza et al., 2018). Hydraulic conditions at the investigated field site remained unclear since flow measurements in PW using heat pulse sensing (e.g. (Lewandowski et al., 2011a)) were unsuccessful. Multi-level temperature lances in the nearby streambed (50 m 
downstream) indicated a downward vertical PW flow (0.06 to $0.11 \mathrm{~m} \mathrm{~h}^{-1}$ (Schaper et al., 2019), 0.12 to $0.89 \mathrm{~m} \mathrm{~d}^{-1}$ (Schaper et al., 2018b)). The same area was identified as a zone of SW exfiltration (approx. $\left.800 \mathrm{~m}^{3} \mathrm{~d}^{-1}\right)($ Verleger and Schumacher, 2012). Therefore, downward vertical PW flow was assumed for the investigated sediment patch. It is however to be considered that sediment characteristics, hydraulic conditions and consequently the hyporheic flow can vary in time and across a small spatial scale, and are difficult to evaluate in general (Angermann et al., 2012).

\subsection{Extraction of passive samplers}

Receiving phases stored at $-20^{\circ} \mathrm{C}$ in acetone were equilibrated to room temperature and then shaken on a rotary shaker in the storage solvent for 30 min. Obtained extracts were transferred to new glass vials and were then spiked with ISs. The receiving phases were extracted a second time using $6 \mathrm{~mL}$ methanol (30 min). The first receiving phase extracts (acetone) were evaporated to roughly $1 \mathrm{~mL}$ using nitrogen blow-down $\left(40^{\circ} \mathrm{C}\right)$ and then combined with the methanolic extract. Combined extracts were filtered $(13 \mathrm{~mm}$ syringe filter, polytetrafluoroethylene, $0.45 \mu \mathrm{m}$ pore size, article no. SF1309-2, BGB analytics, Switzerland), evaporated to 0.1 $\mathrm{mL}$, reconstituted to $1 \mathrm{~mL}$ using NPW, centrifuged (3'020 g for $30 \mathrm{~min}$ at $20^{\circ} \mathrm{C}$, Megafuge $1.0 \mathrm{R}$, Heraeus), and supernatants transferred to sample vials. The final extracts were stored at $4^{\circ} \mathrm{C}$ during immediate subsequent instrumental analysis.

\subsection{LC-HRMS/MS analysis and data processing}

Water grab samples (collected during the uptake experiments, stored at $-20^{\circ} \mathrm{C}$ ) were equilibrated to room temperature and then centrifuged $\left(3,020 \mathrm{~g}\right.$ for $30 \mathrm{~min}$ at $20^{\circ} \mathrm{C}$, Megafuge $1.0 \mathrm{R}$, Heraeus) before supernatant aliquots were transferred into sample vials and spiked with ISs. Calibration standards (details in section 2.1), PS extracts and water samples were injected into a reversed-phase C18 liquid chromatography column (either Atlantis T3, $3 \times 150 \mathrm{~mm}, 3 \mu \mathrm{m}$ or XBridge, $2.1 \times 50 \mathrm{~mm}, 3.5 \mu \mathrm{m}$, both: Waters, USA). NPW and methanol, both acidified with $0.1 \%$ formic acid, were used as eluents for the chromatographic gradient from $5 \%$ (Atlantis T3) or $10 \%$ (XBridge) to $95 \%$ methanol. Chromatography was followed by electrospray ionization and detection by highresolution tandem mass spectrometry (QExactive or QExactive Plus, Thermo Scientific, USA). Mass spectra were acquired in full scan mode at a mass resolution of 140'000 (FWHM at m/z 200) with subsequent data-dependent MS2 (Top5, mass resolution 17'500). Separate runs were carried out in positive and negative ESI polarity mode. More details on the instrumental methods used are provided in the ESM (Table ESM1.A-1). 
Chromatographic peaks of target analytes were automatically detected ( $5 \mathrm{ppm}$ mass tolerance, minimum 3 data points) and integrated using the ICIS algorithm of TraceFinder (version 4.1 EFS, Thermo Scientific, USA). All peak integrations were reviewed manually. Quantification was based on the internal standard method, i.e. to each target analyte, ideally a matching IS was assigned. If the latter was not available, an IS with a similar retention time (nonmatching) was used instead. Linear or quadratic calibration curves $\left(1 / x-\right.$ or $1 / x^{2}$-weighted) were then generated by fitting analyte concentrations $(\mathrm{x})$ against STD-to-IS peak area response ratios $(\mathrm{y})$ without forcing the fit through zero.

\subsection{Suspect screening of hyporheic transformation products}

Raw data of PS3 extracts after field application were submitted to a Compound Discoverer workflow (version 2.1, Thermo Scientific, see section ESM1.K for details) for detection (minimum intensity: 10'000, 30\% intensity tolerance) and grouping (considering different ESI adducts and isotope peaks) of peak features (characterized by $\mathrm{m} / \mathrm{z}$, retention time and intensity) with subsequent compound assignment (elemental composition based on predefined maximum elemental counts within a mass tolerance of $5 \mathrm{ppm}$ ). Compounds were automatically matched with a list containing suspected TP candidates. The latter were phase I metabolites (oxidation, reduction, cleavage), phase II metabolites (conjugates) and several other microbial TPs of selected parent OCs. Metabolites were predicted using a prediction tool that computes metabolites starting from the molecular formula of the parent. For the prediction of further microbial TPs, the Eawag pathway prediction system (Eawag-PPS, http://eawagbbd.ethz.ch) was used, i.e. a web-based tool that employs the SMILES string (molecular structure) of an OC as the input (settings: relative reasoning, no immediate rules, aerobic and anaerobic transformations allowed, 3 generations) to generate predictions for TP pathways. Peak areas of TP suspects in PW were corrected for the difference in flow velocity with respect to SW using the field factor of the parent OC calculated according to Eq. (2). If experimental Rss were unavailable, the average field factor of PS3 (i.e. 2.1) was used instead.

Eq. (2) field factor ${ }_{\mathrm{OC}}=\frac{\text { experimental } \mathrm{Rs}_{\mathrm{OC}} \text { (flowing) }}{\text { experimental } \mathrm{Rs}_{\mathrm{OC}} \text { (static) }}$ 


\section{Results and Discussion}

\subsection{Sampling rates for 144 compounds under stagnant and flowing conditions}

Uptake was evaluated for 144 of the 192 spiked OCs that fulfilled the following conditions: (i) analyzable in water samples and PS extracts, (ii) increase in the receiving phase over time of at least 1 PS configuration, (iii) absence in lab blanks, and (iv) no full depletion (to below LOQ, cf. Table ESM2-1) in the exposure medium.

OC concentrations in the 2 uptake experiments ranged from 151 to $1^{\prime} 025 \mathrm{ng} \mathrm{L}^{-1}$ (aquarium) and 71 to $692 \mathrm{ng} \mathrm{L}^{-1}$ (circular flume). Over the course of 14 days, these levels fluctuated on average by $18 \%$ (aquarium) and $29 \%$ (circular flume). Factors that might have caused the deviation from the intended spike level (approx. $600 \mathrm{ng} \mathrm{L}^{-1}$ ) as well as fluctuations are varying OC levels in dosing solutions (section 2.1), presence of OCs in unspiked tap water (not monitored), sorption-desorption of OCs in the setup materials and abiotic (e.g. hydrolysis) or biotic attenuation (no visual indication of a biofilm). Rss obtained for the different PS configurations under the 2 hydrodynamic conditions are summarized in Table 1 and are also presented in detail in Table ESM2-1.

Table 1 Overview on sampling rates obtained under 2 hydrodynamic conditions for 4 different passive sampler configurations.

\begin{tabular}{|c|c|c|c|c|c|c|c|c|}
\hline $\begin{array}{l}\text { Setup } \\
\text { Flow velocity }\end{array}$ & \multicolumn{4}{|c|}{$\begin{array}{l}\text { Aquarium (stagnant, no renewal) } \\
\text { nearly stagnant (hyporheic zone) }\end{array}$} & \multicolumn{4}{|c|}{$\begin{array}{c}\text { Circular flume (flowing, renewal) } \\
0.13 \mathrm{~m} \mathrm{~s}^{-1} \text { (main channel) }\end{array}$} \\
\hline $\begin{array}{l}\text { Passive sampler } \\
\text { Receiving phase }\end{array}$ & $\begin{array}{c}\boldsymbol{R} \\
\text { SDB }\end{array}$ & $\begin{array}{c}1 \\
\text { SDB }\end{array}$ & 2 & 3 & $\begin{array}{c}\boldsymbol{R} \\
\text { SDB }\end{array}$ & $\begin{array}{c}1 \\
\text { SDB }\end{array}$ & $\begin{array}{c}2 \\
B G\end{array}$ & SDB \\
\hline Uptake limiting barrier(s) & PES & 2 PES & $\begin{array}{l}\text { DG, } \\
\text { PES }\end{array}$ & $\begin{array}{l}\text { DG, } \\
\text { PES }\end{array}$ & PES & 2 PES & $\begin{array}{l}\text { DG, } \\
\text { PES }\end{array}$ & $\begin{array}{l}\text { DG, } \\
\text { PES }\end{array}$ \\
\hline number of $\mathrm{OC}^{\mathrm{a}}$ & 116 & 115 & 117 & 116 & 122 & 109 & 115 & 126 \\
\hline $\begin{array}{ll}\text { Sampling } & \operatorname{mean} \pm S D \\
\text { rate }\left(\mathrm{mL} \mathrm{d}^{-1}\right) & \min . / \max .\end{array}$ & $\begin{array}{l}18 \pm 13 \\
0.3 / 54\end{array}$ & $\begin{array}{l}12 \pm 9 \\
0.3 / 35\end{array}$ & $\begin{array}{c}13 \pm 5 \\
0.4 / 27\end{array}$ & $\begin{array}{c}10 \pm 7 \\
0.2 / 30\end{array}$ & $\begin{array}{c}63 \pm 45 \\
1.4 / 319\end{array}$ & $\begin{array}{c}33 \pm 25 \\
1.5 / 138\end{array}$ & $\begin{array}{l}32 \pm 11 \\
4.4 / 97\end{array}$ & $\begin{array}{l}19 \pm 14 \\
0.7 / 67\end{array}$ \\
\hline
\end{tabular}

Owing to the difference in flow velocity, mean Rss in the circular flume (19 to $63 \mathrm{~mL} \mathrm{~d}^{-1}$ ) were higher than in the aquarium (10 to $18 \mathrm{~mL} \mathrm{~d}^{-1}$ ). As expected, increasing resistance in the uptake limiting barrier resulted in lower Rss (highest for PS-R). However, mean Rss of PS2 and PS3 were different despite the same uptake limiting barrier (diffusion gel covered by PES membrane). This observation can only be explained by better contact between binding and diffusion gel in PS2 compared to SDB-RPS disk and diffusion gel in PS3 or by a higher diffusivity of OCs in the binding gel (PS2) than in the SDB-RPS disk (PS3). PSs with a SDB-RPS disk as the receiving phase (PS1, PS3, PS-R) demonstrated selectivity towards different OC species (Fig. 3), i.e. Rss were low for cations and 
higher for zwitterions, followed by anions and neutral OCs. By contrast, hardly any species discrimination was observed for PS2 with Oasis HLB in the binding gel as the receiving phase. No linear (Pearson) correlation was observed between Rss and $\log \mathrm{Dow}, \mathrm{pH} 8.3$ irrespective of PS-type and uptake experiment. All $\mathrm{r}^{2}$ were below 0.1 except for PS2 under stagnant conditions $\left(r^{2}=0.27, r=-0.52, p<0.005\right)$. After grouping Rss by $O C$ species, this linear correlation between logDow,pH8.3 and Rss in PS2 became more pronounced for neutral OCs $\left(r^{2}=0.44, r=-0.67\right.$, $\mathrm{p}<0.005$ ), i.e. Rss decreased with increasing $\log \mathrm{Dow}_{\mathrm{pH}} \mathrm{s} .3$ (see Fig. ESM1.E-3). In a former study using Chemcatcher in PS-R configuration but with a larger PES membrane pore size $(0.45 \mu \mathrm{m}$, here: $0.10 \mu \mathrm{m})$, universal Rss were derived for very polar anionic (50 mL d-1, here: $67 \mathrm{~mL} \mathrm{~d}^{-1}$ for anions) and semi-polar neutral OCs (100 mL $\mathrm{d}^{-1}$, here: $91 \mathrm{~mL} \mathrm{~d}^{-1}$ for neutral OCs) for flow velocities above $0.05 \mathrm{~m} \mathrm{~s}^{-1}$ (here: $0.13 \mathrm{~m} \mathrm{~s}^{-1}$ ) (Moschet et al., 2015). Based on the observed SDB-RPS disk selectivity, we further suggest universal Rss of $29 \mathrm{~mL} \mathrm{~d}^{-1}$ and $49 \mathrm{~mL} \mathrm{~d}^{-1}$ for cationic and zwitterionic OCs, respectively.
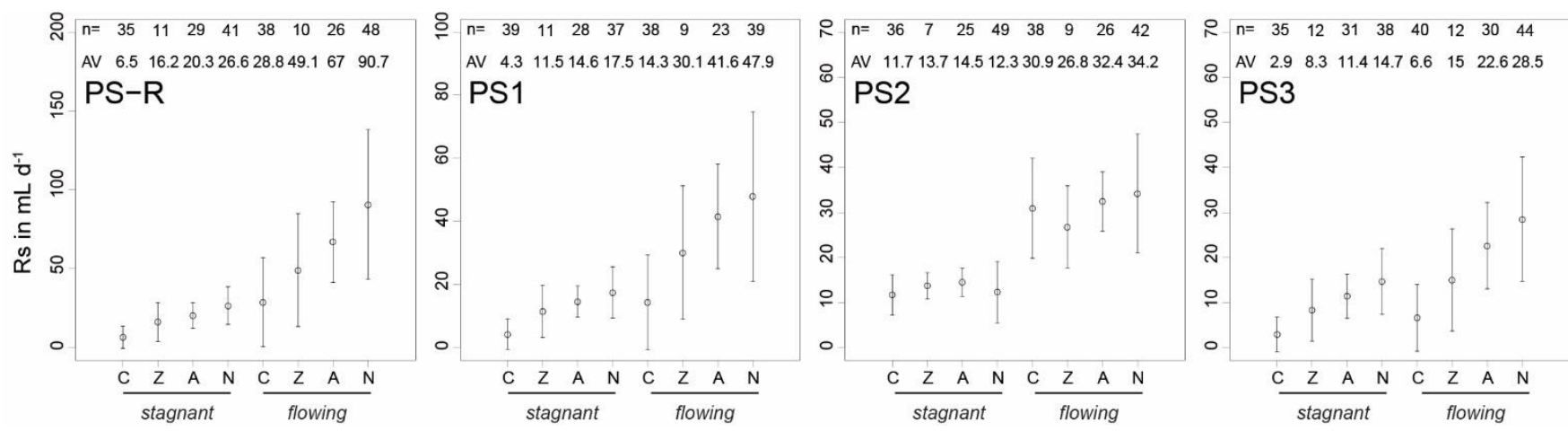

Fig. 3 Influence of speciation on sampling rates (mean Rs \pm standard deviation) for the different PS configurations. Predicted major species at pH 8.3: A: anionic. C: cationic. N: neutral. Z: zwitterionic.

\subsection{The least flow-sensitive passive sampler: SDB-RPS disk-diffusion gel-PES membrane (PS3)}

To identify the most insensitive PS to the tested hydrodynamic conditions, Rss were compared for 81 OCs with demonstrated linear uptake by all 4 PSs in both experiments. OC-specific differences between Rss $(\Delta$ Rs) were calculated according to equation Eq. (3) and are summarized in Table 2.

Eq. (3) $\Delta \mathrm{Rs}_{\mathrm{OC}}$ in $\%=\left|\left(\frac{\mathrm{Rs}_{\mathrm{OC}} \text { (flowing) }}{\mathrm{Rs}_{\mathrm{OC}} \text { (static) }} \times 100 \%\right)-100 \%\right|$ 
Table 2 Sensitivity of tested passive samplers towards hydrodynamic conditions (flow velocity)

expressed as difference in sampling rates $(\Delta \mathrm{Rs})$.

\begin{tabular}{rcccc}
\hline Passive sampler & $\boldsymbol{R}$ & $\mathbf{1}$ & $\mathbf{2}$ & $\mathbf{3}$ \\
Receiving phase & SDB & SDB & BG & SDB \\
Uptake limiting barrier(s) & PES & 2 PES & DG, PES & DG, PES \\
\hline Mean \% $\%$ Rs & 274 & 235 & 147 & 110 \\
Median \% $\Delta$ Rs & 275 & 228 & 133 & 98 \\
Minimum \% $\Delta$ Rs & 96 & 92 & 3 & 45 \\
Maximum \% $\Delta R s$ & 564 & 553 & 484 & 303 \\
\hline Abbreviations: SDB: SDB-RPS disk, BG: binding gel, DG: diffusion gel, PES: polyethersulfone membrane
\end{tabular}

None of the investigated PSs was entirely insensitive to flow velocity. However, PS3, a combination of Chemcatcher (receiving phase) and o-DGT (diffusion gel) was the least sensitive to flow velocity for the tested OCs, i.e. the mean $\Delta$ Rs was smallest with $110 \% . \Delta$ Rs was the highest in the PS-R (274\%), followed by PS1 (235\%) and PS2 (a modified o-DGT, 147\%). A similar $\Delta$ Rs (approx. 100\%) was reported for uptake of the antibiotic sulfamethoxazole into an o-DGT, when the stirring speed was increased from 0 to a high value (Chen et al., 2012). As expected, the PS-R without any modifications was the most sensitive to hydrodynamics. Looking closely at the individual PS configurations revealed a significant positive correlation $(r \geq 0.27, p<0.05)$ between $\Delta$ Rs and logDow,pH8.3 for all PSs except PS3 ( $r=0.20, p>0.05)$. Roughly speaking, less polar analytes were more affected by the different flow conditions. Depending on the PS configuration, this correlation was even more pronounced at the speciation level, e.g. the correlation between $\Delta$ Rs and $\log D_{0}$,pH8.3 of anions in PS2 $(r=0.7, p<0.005)$. Finally, PS3 was deemed as the most applicable PS for the main channel and $\mathrm{HZ}$ of the Erpe because it was the least sensitive to the changes in water flow velocity and the receiving phase was physically more stable, allowing for easier handling than the binding gel of PS2.

\subsection{Quantification of 104 organic contaminants across the water-sediment interface of the Erpe}

A total of 104 OCs (81 parent OCs, 23 TPs) were quantified above LOQ when PS3 were deployed in the Erpe. Of the detected compounds, 68 were pharmaceuticals (21 TPs), 4 illicit drugs, 3 biocides (1 TP), 2 corrosion inhibitors (1 TP), 2 industrial chemicals, 1 personal care product and $1 \mathrm{X}$-ray contrast agent (classification based on major use in Europe). Stringent requirements for the evaluation of an $\mathrm{OC}$ was its absence in field blanks, requirement for the evaluation of an OC in SW was its presence in all 3 PS replicates. The latter criterion was not applied to PW PSs since they were not necessarily exposed to the same PW or hyporheic flow path. Moreover, scouring was observed around holder 1 (most upstream). Hence the PS at $-0.15 \mathrm{~m}$ (only holder 1 ) was excluded from the trend analyses. Overall, the OC mass among PS replicates in SW varied by $10 \%$, indicating the robustness of the PS 
method. In PW, the OC mass per PS was averaged for the 3 PSs at a specific depth. These levels underwent moderately higher fluctuations (20 to $27 \% \mathrm{RSD}$ ) in the underlying layer ( $\mathrm{HZ}$ ), likely suggesting that the sediment characteristics were not entirely homogenous around the different holders. Furthermore, a horizontal PW flow component was ruled out, which is in agreement with 2 earlier studies at the Erpe, where no significant horizontal PW flow was observed (Posselt et al., 2018; Schaper et al., 2018b). The TWA concentrations were derived using Eq. (1) with the OC mass per PS and the Rs as inputs. In the best case, experimental Rss were available for both flow scenarios (stagnant, $n=71$, flowing, $n=74$ ). If the Rs was only available for one of the 2 scenarios, the missing Rs was estimated by linear correlation between Rss of PS3 in the 2 setups (stagnant, $n=5$, flowing, $n=2$, see Fig. ESM1.G-5 for details). Whenever no experimental Rs could be determined the OC species-specific (stagnant, $\mathrm{n}=20$, flowing, $n=18$, Fig. 3) or setup-specific Rs (mean Rs of all OCs, stagnant: $9.6 \mathrm{~mL} \mathrm{~d}^{-1}, \mathrm{n}=8$, flowing: $18.9 \mathrm{~mL}$ $d^{-1}, n=10$ ) was used. The final error of $O C$ concentrations in water (error bars in concentration profiles, Fig. 4) ranged from 5 to $120 \%$ and resulted from error propagation taking into account the Rs error (stagnant: 5 to $69 \%$, flowing: 10 to 47\%) and the deviation of OC mass among PS replicates (see above).

\subsubsection{OC concentrations in surface water}

All 104 OCs above LOQ were present in SW at TWA concentrations ranging between $3.1 \pm 0.7 \mathrm{ng} \mathrm{L}^{-1}$ (iminostilbene) and $29 \pm 7.2 \mu \mathrm{g} \mathrm{L}-1$ (melamine). Besides melamine, 17 other parent OCs were found in the $\mu \mathrm{g} / \mathrm{L}-$ range, such as valsartan $\left(23 \pm 4.9 \mu \mathrm{g} \mathrm{L}^{-1}\right), 1 \mathrm{H}$-benzotriazole $\left(8.1 \pm 2.5 \mu \mathrm{g} \mathrm{L}^{-1}\right)$, sitagliptin $\left(6.8 \pm 1.6 \mu \mathrm{g} \mathrm{L}^{-1}\right)$, metoprolol $\left(6.4 \pm 1.3 \mu \mathrm{g} \mathrm{L}^{-1}\right)$, hydrochlorothiazide $\left(5.3 \pm 1.2 \mu \mathrm{g} \mathrm{L}^{-1}\right)$, metformin $\left(4.2 \pm 1.2 \mu \mathrm{g} \mathrm{L}^{-1}\right)$, diclofenac $\left(3.9 \pm 0.8 \mu \mathrm{g} \mathrm{L}^{-1}\right)$, gabapentin $(3.4 \pm 0.8 \mu \mathrm{g}$ $\left.\mathrm{L}^{-1}\right)$ and 5-methyl-1H-benzotriazole $\left(2.9 \pm 0.9 \mu \mathrm{g} \mathrm{L}^{-1}\right)$ (see Table ESM2-1 for all OCs). These elevated concentrations can be explained by the high proportion of STP effluent (up to 80\%) that Erpe receives from upstream STPs. Similar concentrations were reported in a parallel study over 2 days (June 14 to June 17, 2016) based on an hourly sampling resolution (comparison provided in Fig. ESM1.I-8) (Jaeger et al., 2019).

\subsubsection{OC concentration changes across the water-sediment interface}

To evaluate OC concentration changes across the water-sediment interface, TWA concentrations in SW were compared with TWA concentrations in PW. By doing so, 3 distinct vertical concentration patterns were identified (Table 3). Example compounds are depicted in Fig. 4. Pattern 1 (attenuation) can be further divided into 4 subpatterns (1a to $1 \mathrm{~d}$ ). Since PW was anoxic after approx. $-0.30 \mathrm{~m}$ (section 2.5), only little formation of aerobic TPs was expected below this depth. Moreover, due to the elevated fraction of sediment organic carbon, sorptive 
attenuation of especially apolar OCs was likely. Under such conditions, it is essential to link parent attenuation to TP formation. Of the 104 OCs, 30 OCs (14 parents, 16 TPs) could be assigned to 10 groups of at least one parent OC and one known TP. Some of them will be discussed in detail below. The other 73 OCs were either parent OCs without monitored TP $(n=67)$ or TPs without detected parent OC $(n=6$, parent below LOQ: irgarol, nicotine, verapamil, analytical issue: tramadol, not monitored: fenofibrate).

Table 3. Concentration patterns of organic contaminants $(\mathrm{OC})$ across the water-sediment interface.

\begin{tabular}{|c|c|c|c|c|c|c|c|c|}
\hline \multirow{3}{*}{$\begin{array}{l}\text { (Sub) } \\
\text { Group }\end{array}$} & \multicolumn{3}{|c|}{ Pattern description } & \multicolumn{5}{|c|}{ Substance characteristics } \\
\hline & \multirow{2}{*}{$\begin{array}{l}\text { Interface } \\
\text { SW to PW (-0.15 } \\
\text { m) }\end{array}$} & \multirow{2}{*}{$\begin{array}{l}\text { Porewater } \\
-0.15 \text { to }-0.45 m\end{array}$} & \multirow{2}{*}{$\# O C$} & \multicolumn{4}{|c|}{ Speciation $_{p H 7.9}$} & \multirow{2}{*}{$\begin{array}{c}\log D_{\text {ow,pH7.9 }} \\
\text { mean }\end{array}$} \\
\hline & & & & $N$ & $C$ & $A$ & $z$ & \\
\hline \multirow{4}{*}{1} & \multirow{4}{*}{ attenuation $^{a}$} & $<\mathrm{LOQ}$ & 33 & 13 & 15 & 3 & 2 & 1.4 \\
\hline & & $\begin{array}{l}\text { linear increase } \\
\left(r^{2} \geq 0.75\right)\end{array}$ & 37 & 14 & 6 & 14 & 3 & 0.5 \\
\hline & & constant ${ }^{\mathrm{b}}$ & 25 & 11 & 8 & 6 & 0 & 0.5 \\
\hline & & other (fluctuations) & 5 & 1 & 3 & 1 & 0 & 1.5 \\
\hline 2 & \multicolumn{2}{|c|}{$\begin{array}{l}\text { linear increase }\left(r^{2}>0.95\right) \text { from SW to }-0.45 \\
m\end{array}$} & 1 & 0 & 0 & 1 & 0 & -1.7 \\
\hline 3 & \multicolumn{2}{|c|}{ constant $^{\mathrm{b}}(\leq 20 \%)$ from SW to $-0.45 \mathrm{~m}$} & 3 & 2 & 1 & 0 & 0 & 1.6 \\
\hline
\end{tabular}




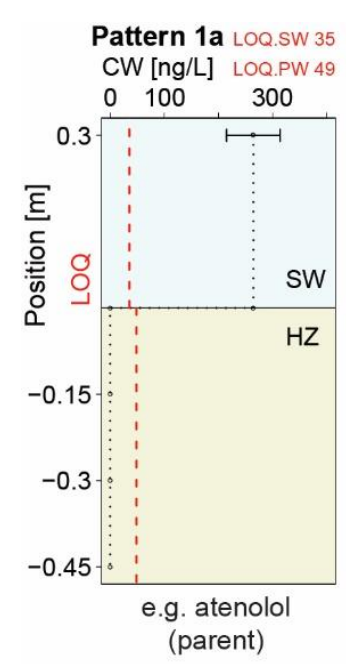

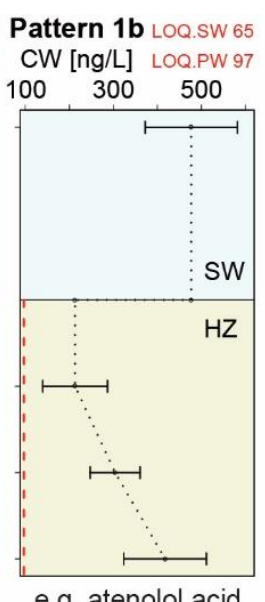

(TP)

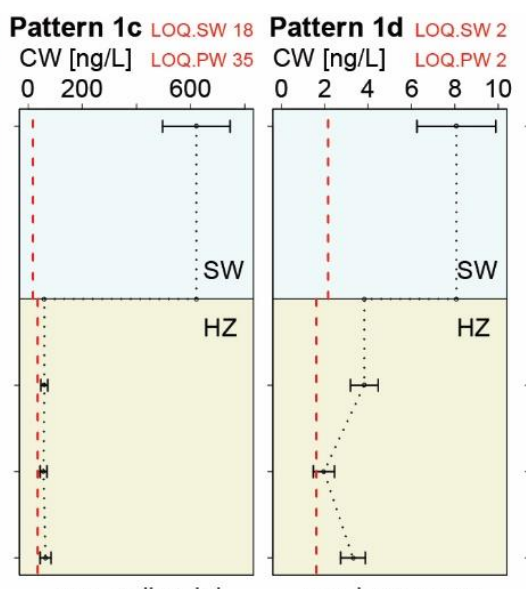

e.g. celiprolol (parent)

Pattern 2 LOQ.SW 2 Pattern 3 LOQ.SW 2 $\mathrm{CW}[\mu \mathrm{g} / \mathrm{L}] \quad$ LOQ.PW $0.4 \mathrm{CW}[\mathrm{ng} / \mathrm{L}] \quad$ LOQ.PW 3

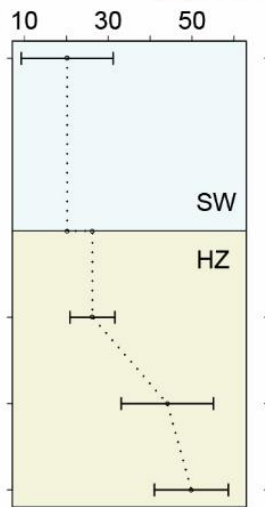

e.g. valsartan acid (TP)

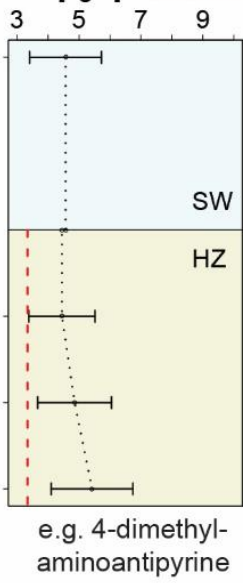

(parent)

Fig. 4. Concentration patterns across the water-sediment interface exemplarily shown for 6 organic contaminants.

Red dashed lines: limit of quantification (LOQ) in the respective compartment (value in in $\mathrm{ng} \mathrm{L}^{-1}$ in top margin).

Black dotted line: linear interpolation between mean concentrations (standard deviation as error bars).

Concentrations below LOQ are plotted as $0 \mathrm{ng} / \mathrm{L}$. Abbreviations: CW: concentration in water. HZ: hyporheic zone.

PW: porewater. SW: surface water. TP: transformation product.

Not all 104 OCs present in SW were also found in PW. Specifically, 33 OCs (24 parents, 9 TPs) were exclusively found in SW ( $<L O Q$ in PW) (Table 3, pattern 1a) and can be considered to have been completely attenuated in the water-sediment interface (SW to PW at $-0.15 \mathrm{~m}$ ). To account for different LOQs in SW and PW, the PW LOQ was used for calculation of the degree of attenuation. This resulted in a strong attenuation for most OCs ( $n=25,24$ to $94 \%)$ whereas for some $(n=5)$ the change was not significant $(<20 \%)$. Because most OCs in pattern 1a were semipolar or relatively apolar $\left(n=19, \log \mathrm{D}_{\mathrm{ow}, \mathrm{pH7}} \mathrm{n} 1.2\right.$ to 4.6$)$ and neutral $(n=13)$ or cationic $(n=15)$, some sorption is expected in the organic-rich sediment (section 2.5). At the same time, biodegradation in the $\mathrm{HZ}$ is also a likely process, since several OCs in pattern 1a are known to be biodegradable in STPs (e.g. atenolol, citalopram, fexofenadine, ranitidine, telmisartan, trimethoprim) (Otto et al., 2014).

Overall, 37 OCs (29 parents, 8 TPs) demonstrated attenuation from SW to PW and subsequent linear increase with increasing PW depth without exceeding the TWA concentration in SW (pattern 1b). The concentration increase of these substances with PW depth suggests that the $\mathrm{HZ}$ acts as sink or source of $\mathrm{OC}$, depending on the underlying hydraulic conditions. This observation is in agreement with previous observations based on PW sampling at high spatial and temporal resolution (Posselt et al., 2018). Most OC in pattern $1 \mathrm{~b}(\mathrm{n}=26)$ were polar $\left(\log \mathrm{D}_{\mathrm{ow}, \mathrm{pH}} \mathrm{g} .9 \leq 1\right)$ but of diverse speciation. Thus, substance properties alone cannot explain this phenomenon. However, it can be speculated that due to the low flow velocity and eutrophic conditions in the Erpe (Lewandowski et al., 2011b), the 
highly organic top sediment layer (section 2.5) served as sorption site for many OCs at $-0.15 \mathrm{~m}$. By contrast, elevated concentrations of many $\mathrm{OC}$ at $-0.45 \mathrm{~m}$ could have originated from eventual connectivity to a highly contaminated groundwater body. Note that a detailed hydrological analysis was not completed within this study, so caution must be exercised when interpreting the results. For 2 parent OCs which were attenuated to below LOQ (pattern 1a), the monitored TP followed pattern 1b (increase with increasing PW depth). The first TP was clopidogrel carboxylic acid, a major human metabolite of clopidogrel formed by enzymatic hydrolysis (esterase). The second TP was atenolol acid/metoprolol acid formed from atenolol by (microbial) enzymatic hydrolysis (Radjenović et al., 2008) and from metoprolol through CYP450-mediated dealkylation during aerobic microbial biotransformation or in human metabolism (Kern et al., 2010; Rubirola et al., 2014). Both transformations appear feasible in the oxic (0 to $-0.30 \mathrm{~m}$ ) and anoxic zones (below $-0.30 \mathrm{~m}$ ) of Erpe PW, except for an aerobic biodegradation of metoprolol in deep PW (below $-0.30 \mathrm{~m})$. Since both TP classify as polar $\left(\log \mathrm{D}_{\mathrm{ow}, \mathrm{pH} .9} \leq 1\right)$ and carry a negative charge at field $\mathrm{pH}$, high subsurface mobility could be expected. Thus, elevated concentrations in PW could have resulted from emission by upstream STP with subsequent transport into PW (especially since SW concentrations were exceeding PW concentrations), but also from in-situ formation in the $\mathrm{HZ}$.

The increasing concentration from SW to $-0.45 \mathrm{~m}$ in PW was only observed for valsartan acid (pattern 2, Fig. 4). In fact, the concentration already exceeded the SW concentration at $-0.15 \mathrm{~m}$. Valsartan acid is a polar $\left(\log \mathrm{D}_{\mathrm{ow}, \mathrm{pH}} \mathrm{H.9,-}\right.$ 1.7) and anionic TP of valsartan (Helbling et al., 2010; Kern et al., 2010) and conceivably other structurally related antihypertensive drugs, such as candesartan ( $\log \mathrm{D}_{\mathrm{ow}, \mathrm{pH}} \mathrm{7.9}, 0.3$, anionic), irbesartan $\left(\log \mathrm{D}_{\mathrm{ow}, \mathrm{pH} 7.9}, 4.1\right.$, anionic), losartan (log $\mathrm{D}_{\mathrm{ow}, \mathrm{pH} 7.9}, 3.6$, anionic) and olmesarten $\left(\log \mathrm{D}_{\mathrm{ow}, \mathrm{pH} 7.9,}-1.2\right.$, anionic, not analyzed) (concentration profiles in section ESM1.J) (Zirlewagen et al., 2013). Although its formation from valsartan (log $\mathrm{D}_{\mathrm{ow}, \mathrm{pH}} \mathrm{H.9}, 0.6$, anionic) is known for wastewater treatment and activated sludge (Helbling et al., 2010), the occurrence in sediment PW has only recently been reported for the Erpe (Posselt et al., 2018; Schaper et al., 2019). Concentrations obtained by active sampling in the latter studies and measured PS concentrations differ by an order of magnitude (comparison provided in Fig. ESM1.I-9). The latter is likely - active and passive sampling periods were barely overlapping, meaning that PS were presumably exposed to different OC concentrations and fluctuations caused by the upstream STP. Another explanation could be an incorrect Rs estimate for valsartan acid. The Rs for this compound could not be determined in the uptake experiments over 14 days as valsartan acid equilibrated with the receiving phase (SDB-RPS disk) in <2 days of exposure. The mean partition coefficient of valsartan acid between SDB-RPS disk and tap water at assumed equilibrium $\left(\mathrm{KSDBw}=\mathrm{mSDB} \times \mathrm{CW}^{-1}\right)$ for days 6 and 14 accounted for $4.6 \pm 0.3 \mathrm{~mL}$ SDBRPS disk ${ }^{-1}$ (40 mm cutout) under stagnant and 3.2 $\pm 0.7 \mathrm{~mL} \mathrm{SDB}-R P S$ disk $^{-1}$ under flowing conditions. Rss calculated 
for the 2 day-exposure of PS3 were $2.2 \pm 0.3 \mathrm{~mL} \mathrm{~d}^{-1}$ (stagnant) and $1.6 \pm 0.6 \mathrm{~mL} \mathrm{~d}^{-1}$ (flowing). For comparison, the anion-specific Rs of PS3 under stagnant and flowing conditions accounted for 11 and $23 \mathrm{~mL} \mathrm{~d}^{-1}$, respectively (section 3.1). PS PW concentrations of valsartan acid should therefore be considered as TWA of the (maximum) 2 days before retrieval or as (equilibrium) concentration snapshot at the time of retrieval. Using either of the 2 assumptions or the species-specific Rs, the trend for valsartan acid remains the same (pattern 2).

Among the monitored sartans, valsartan was the most dominant sartan in SW. Its transformation to valsartan acid involves 3 steps: (1) oxidative dealkylation of valsartan at the tertiary amide group, (2) hydrolysis to amino-valsartan at the secondary amide group, and (3) oxidative deamination to valsartan acid (Helbling et al., 2010; Kern et al., 2010). Because some steps require oxic conditions, the formation in upper PW ( -0.15 to $-0.30 \mathrm{~m})$ and accumulation in anoxic PW (-0.45 m) likely contributed to what was observed in Fig. 4. As iterated before, the HZ may act as sink or source for this TP, depending on the underlying hydraulic conditions.

\subsection{Screening for further transformation products}

For 49 selected parent $\mathrm{OC}$ with a minimum TWA concentration of $50 \mathrm{ng} \mathrm{L}^{-1}$ in SW and a minimum attenuation of $63 \%$ at the water-sediment interface (34\% if the PW LOQ was accounted for), TP suspects were predicted using metabolic logic (section 2.8) and screened for in PS extracts. After peak areas in PW were corrected for the flow velocity difference compared to SW using a field factor (section 2.8), total potential TP signals across the watersediment interface (214'270 in positive and 138'714 in negative ESI mode) were filtered (peak area cut-off, field blank, exclusion of STD and IS) and submitted to hierarchical cluster analysis ('hclust' in the 'cluster' R package) (Chiaia-Hernandez et al., 2017). Of particular interest were patterns hinting at increased formation in the HZ (Fig.

4, pattern 2). Compounds following this pattern were reviewed for retention time plausibility using the linear relationship $\left(r^{2}=0.67\right)$ between predicted logDow (at mobile phase $\left.\mathrm{pH} 3\right)$ and STD retention times $(n=590)$ from literature for the same liquid chromatographic system (predicted retention time $=($ predicted $\operatorname{logDow}, \mathrm{pH} 3+5.4) \times$ $0.42^{-1} \mathrm{~min}$ ) (Mechelke et al., 2019). In case of duplicates at different retention times, only the most intense compound was considered (resulting in $n=802$ TP suspects). After the chromatography was visually inspected $(n=381)$, TP candidates predicted from the molecular formula of the parent OC were reasoned $(n=127)$. Compounds were only further inspected if a data-dependent MS2 was triggered $(n=28)$. The obtained MS2 spectra were compared with in-silico predicted MS2 fragments and annotated using the Compound Discoverer Fragment Ion Search (FISh). The final candidates $(n=9)$ featuring an isotope pattern match (spectral fit) between $53 \%$ and $97 \%$ and a FISh score $>0 \%$ are presented in section ESM1.L. The most likely candidate was N-[4-(2- 
hydroxyacetyl)phenyl]methanesulfonamide (CAS 287944-24-5, C9H11NO4S, monoisotopic mass: 229.0409 Da), a TP suspect of the beta blocker sotalol predicted via the Eawag pathway prediction system (rule bt0063: secondary amine to amine and aldehyde, Sot-disnCHO) and detected in negative and positive ESI mode. A structurally similar TP of sotalol (N-(4-formylphenyl) methanesulfonamide) has been found in a former but not in the present study (Stadlmair et al., 2019). In conclusion, several potential TPs followed pattern 2, however, many were lacking structural evidence, as e.g. diagnostic MS2 fragments or unequivocal halogen isotopic patterns.

\section{Conclusions}

- Two uptake experiments provided Rss for 4 PS configurations under stagnant and flowing conditions and for a broad set of OCs, many among them being frequently detected in water bodies. Application of these Rss to other low-flow scenarios, such as groundwater wells is possible.

- Increasing solute transfer resistance in the uptake limiting barrier did not render the tested PS configurations entirely insensitive towards changing hydrodynamic conditions. However, sensitivity could be considerably reduced compared to the unmodified reference PS (a Chemcatcher with a PES membrane and SDB-RPS receiving phase).

- PSs consisting of SDB-RPS disk as the receiving phase demonstrated discrimination of anionic, cationic, zwitterionic and neutral OC species. This discrimination was only minor in the tested agarose gel with Oasis HLB, making the estimation of $\mathrm{OC}$ concentrations in water more robust. However, since the binding gel was very fragile and proved difficult to handle, the SDB-RPS disk was preferred over the gel for the application in the field.

- Passive sampling across the water-sediment interface allowed for the determination of $104 \mathrm{OC}$ concentration profiles at a STP effluent-dominated urban stream (Erpe), causing minimum sediment disturbance and only requiring minimal supervision. Comparison with concentration data at high spatial and temporal resolution in SW and in the PW of a nearby sediment patch revealed that PS concentrations (11-day average) were often a good approximation of concentrations (2-day average) obtained by active sampling in another study.

- Various spatial trends across the water-sediment interface were identified. In particular, the formation of valsartan acid from valsartan and conceivably other structurally related antihypertensive drugs in the $\mathrm{HZ}$ was observed. 
- Uncertainties in water paths, sediment and PW characteristics limited the differentiation of fate processes and identification of TPs. A more detailed characterization of sites and a combination of different $\mathrm{HZ}$ sampling approaches might facilitate the characterization of fate processes in the field in the future.

\section{Acknowledgements}

Our special thanks go to Andrea Betterle (W\&T, Eawag) for support with flow velocity measurements in the field, Anna Jäger (IGB, Berlin) for executing the passive sampling screening at the Erpe and help in the field with sediment passive samplers, Anne Mehrtens (IBU, Carl von Ossietzky University of Oldenburg) for taking saturated hydraulic conductivity measurements and support in the field, Malte Posselt (ACES, Stockholm University) for performing oxygen measurements in sediment cores, Muhammad Raza (IAG, TU Darmstadt) for providing data on the sediment organic carbon fraction at the Erpe and all other HypoTRAIN partners for excellent collaboration at the field site. Furthermore, we thank the Eawag workshop team for manufacturing passive sampler parts and components of the field installation device, Prof. Pablo A. Lara-Martin (Universidad de Cádiz) for support with the hierarchical cluster analysis, Maricor Arlos (Uchem, Eawag) for proofreading the manuscript, and Haijia Zeng (University of Zurich) for contributions to the data analysis in the context of his semester project. Finally, we gratefully acknowledge ChemAxon Ltd. for the donation of the academic research license to the JChem package.

\section{Funding}

This project has received funding from the European Union's Horizon 2020 research and innovation programme under the Marie Sklodowska-Curie grant agreement No 641939 and the Swiss State Secretariat for Education, Research and Innovation.

\section{Conflict of interest}

The authors declare that they have no known competing financial interests or personal relationships that could have appeared to influence the work reported in this paper. 


\section{References}

Angermann, L., Lewandowski, J., Fleckenstein, J.H., Nützmann, G., 2012. A 3D analysis algorithm to improve interpretation of heat pulse sensor results for the determination of small-scale flow directions and velocities in the hyporheic zone. J. Hydrol. 475, 1-11. https://doi.org/10.1016/j.jhydrol.2012.06.050

Booij, K., Chen, S., 2018. Review of atrazine sampling by polar organic chemical integrative samplers and Chemcatcher. Environ. Toxicol. Chem. 37, 1786-1798. https://doi.org/10.1002/etc.4160

Bopp, S., Weiß, H., Schirmer, K., 2005. Time-integrated monitoring of polycyclic aromatic hydrocarbons (PAHs) in groundwater using the Ceramic Dosimeter passive sampling device. J. Chromatogr. A 1072, 137-147. https://doi.org/10.1016/j.chroma.2004.12.049

Boulton, A.J., Findlay, S., Marmonier, P., Stanley, E.H., Valett, H.M., 2002. The Functional Significance of the Hyporheic Zone in Streams and Rivers. Annu. Rev. Ecol. Syst. 29, 59-81. https://doi.org/10.1146/annurev.ecolsys.29.1.59

Burke, V., Greskowiak, J., Asmuß, T., Bremermann, R., Taute, T., Massmann, G., 2014. Temperature dependent redox zonation and attenuation of wastewater-derived organic micropollutants in the hyporheic zone. Sci. Total Environ. 482-483, 53-61. https://doi.org/10.1016/j.scitotenv.2014.02.098

Challis, J.K., Hanson, M.L., Wong, C.S., 2016. Development and Calibration of an Organic-Diffusive Gradients in Thin Films Aquatic Passive Sampler for a Diverse Suite of Polar Organic Contaminants. Anal. Chem. 88, 10583-10591. https://doi.org/10.1021/acs.analchem.6b02749

Chen, C.E., Jones, K.C., Ying, G.G., Zhang, H., 2014. Desorption kinetics of sulfonamide and trimethoprim antibiotics in soils assessed with diffusive gradients in thin-films. Environ. Sci. Technol. 48, 5530-5536. https://doi.org/10.1021/es500194f

Chen, C.E., Zhang, H., Jones, K.C., 2012. A novel passive water sampler for in situ sampling of antibiotics. J. Environ. Monit. 14, 1523-1530. https://doi.org/10.1039/c2em30091e

Chen, C.E., Zhang, H., Ying, G.G., Jones, K.C., 2013. Evidence and recommendations to support the use of a novel passive water sampler to quantify antibiotics in wastewaters. Environ. Sci. Technol. 47, 13587-13593. https://doi.org/10.1021/es402662g

Chen, W., Li, Y., Chen, C.E., Sweetman, A.J., Zhang, H., Jones, K.C., 2017. DGT Passive Sampling for Quantitative in Situ Measurements of Compounds from Household and Personal Care Products in Waters. Environ. Sci. 
Technol. 51, 13274-13281. https://doi.org/10.1021/acs.est.7b03940

Chen, W., Pan, S., Cheng, H., Sweetman, A.J., Zhang, H., Jones, K.C., 2018. Diffusive gradients in thin-films (DGT) for in situ sampling of selected endocrine disrupting chemicals (EDCs) in waters. Water Res. 137, 211-219. https://doi.org/10.1016/j.watres.2018.03.029

Chiaia-Hernandez, A.C., Günthardt, B.F., Frey, M.P., Hollender, J., 2017. Unravelling contaminants in the anthropocene using statistical analysis of liquid chromatography-high-resolution mass spectrometry nontarget screening data recorded in lake sediments. Environ. Sci. Technol. 51, 12547-12556. https://doi.org/10.1021/acs.est.7b03357

Cristale, J., Katsoyiannis, A., Chen, C., Jones, K.C., Lacorte, S., 2013. Assessment of flame retardants in river water using a ceramic dosimeter passive sampler. Environ. Pollut. 172, 163-169. https://doi.org/10.1016/j.envpol.2012.08.014

Davison, W., Zhang, H., 1994. In situ speciation measurements of trace components in natural waters using thinfilm gels. Nature 367, 546-548. https://doi.org/10.1038/367546a0

Duff, J.H., Murphy, F., Fuller, C.C., Triska, F.J., Harvey, J.W., P, A., 2007. A Mini Drivepoint Sampler for Measuring Pore Water Solute Concentrations in the Hyporheic Zone of Sand-Bottom Streams. Limnology 43, 13781383.

Gruzalski, J.G., Markwiese, J.T., Carriker, N.E., Rogers, W.J., Vitale, R.J., Thal, D.I., 2016. Pore water collection, analysis and evolution: The need for standardization, in: de Voogt, W.P. (Ed.), Reviews of Environmental Contamination and Toxicology. Springer International Publishing, Cham, pp. 37-51. https://doi.org/10.1007/978-3-319-23573-8_2

Guan, D.X., Li, Y.Q., Yu, N.Y., Yu, G.H., Wei, S., Zhang, H., Davison, W., Cui, X.Y., Ma, L.Q., Luo, J., 2018. In situ measurement of perfluoroalkyl substances in aquatic systems using diffusive gradients in thin-films technique. Water Res. 144, 162-171. https://doi.org/10.1016/j.watres.2018.07.031

Gücker, B., Brauns, M., Pusch, M.T., 2006. Effects of wastewater treatment plant discharge on ecosystem structure and function of lowland streams. J. North Am. Benthol. Soc. 25, 313-329. https://doi.org/10.1899/08873593(2006)25[313:eowtpd]2.0.co;2

Gücker, B., Pusch, M.T., 2006. Regulation of nutrient uptake in eutrophic lowland streams. Limnol. Oceanogr. 51, 1443-1453. https://doi.org/10.4319/lo.2006.51.3.1443 
Guibal, R., Buzier, R., Charriau, A., Lissalde, S., Guibaud, G., 2017. Passive sampling of anionic pesticides using the Diffusive Gradients in Thin films technique (DGT). Anal. Chim. Acta 966, 1-10. https://doi.org/10.1016/j.aca.2017.02.007

Guo, C., Zhang, T., Hou, S., Lv, J., Zhang, Y., Wu, F., Hua, Z., Meng, W., Zhang, H., Xu, J., 2017. Investigation and Application of a New Passive Sampling Technique for in Situ Monitoring of Illicit Drugs in Waste Waters and Rivers. Environ. Sci. Technol. 51, 9101-9108. https://doi.org/10.1021/acs.est.7b00731

Helbling, D.E., Hollender, J., Kohler, H.-P.E., Singer, H., Fenner, K., 2010. High-Throughput Identification of Microbial Transformation Products of Organic Micropollutants. Environ. Sci. Technol. 44, 6621-6627. https://doi.org/10.1021/es100970m

Jaeger, A., Posselt, M., Betterle, A., Schaper, J., Mechelke, J., Coll, C., Lewandowski, J., 2019. Spatial and Temporal Variability in Attenuation of Polar Organic Micropollutants in an Urban Lowland Stream. Environ. Sci. Technol. 53, 2383-2395. https://doi.org/10.1021/acs.est.8b05488

Kern, S., Baumgartner, R., Helbling, D.E., Hollender, J., Singer, H., Loos, M.J., Schwarzenbach, R.P., Fenner, K., 2010. A tiered procedure for assessing the formation of biotransformation products of pharmaceuticals and biocides during activated sludge treatment. J. Environ. Monit. 12, 2100-2111. https://doi.org/10.1039/c0em00238k

Krom, M.D., Davison, P., Zhang, H., Davison, W., 1994. High-resolution pore-water sampling with a gel sampler. Limnol. Oceanogr. 39, 1967-1972. https://doi.org/10.4319/lo.1994.39.8.1967

Kunkel, U., Radke, M., 2012. Fate of pharmaceuticals in rivers: Deriving a benchmark dataset at favorable attenuation conditions. Water Res. 46, 5551-5565. https://doi.org/10.1016/j.watres.2012.07.033

Kunkel, U., Radke, M., 2011. Reactive tracer test to evaluate the fate of pharmaceuticals in rivers. Environ. Sci. Technol. 45, 6296-6302. https://doi.org/10.1021/es104320n

Kunkel, U., Radke, M., 2008. Biodegradation of acidic pharmaceuticals in bed sediments: Insight from a laboratory experiment. Environ. Sci. Technol. 42, 7273-7279. https://doi.org/10.1021/es801562j

Lewandowski, J., Angermann, L., Nützmann, G., Fleckenstein, J.H., 2011a. A heat pulse technique for the determination of small-scale flow directions and flow velocities in the streambed of sand-bed streams. Hydrol. Process. 25, 3244-3255. https://doi.org/10.1002/hyp.8062

Lewandowski, J., Putschew, A., Schwesig, D., Neumann, C., Radke, M., 2011b. Fate of organic micropollutants in 
the hyporheic zone of a eutrophic lowland stream: Results of a preliminary field study. Sci. Total Environ. 409, 1824-1835. https://doi.org/10.1016/j.scitotenv.2011.01.028

Li, Z., Sobek, A., Radke, M., 2015. Flume experiments to investigate the environmental fate of pharmaceuticals and their transformation products in streams. Environ. Sci. Technol. 49, 6009-6017. https://doi.org/10.1021/acs.est.5b00273

Liu, H.H., Bao, L.J., Feng, W.H., Xu, S.P., Wu, F.C., Zeng, E.Y., 2013. A multisection passive sampler for measuring sediment porewater profile of dichlorodiphenyltrichloroethane and its metabolites. Anal. Chem. 85, 71177124. https://doi.org/10.1021/ac400589a

Lydy, M.J., Landrum, P.F., Oen, A.M., Allinson, M., Smedes, F., Harwood, A.D., Li, H., Maruya, K. a, Liu, J., 2014. Passive sampling methods for contaminated sediments: state of the science for organic contaminants. Integr. Environ. Assess. Manag. 10, 167-78. https://doi.org/10.1002/ieam.1503

Malard, F., Tockner, K., Dole-Olivier, M.J., Ward, J. V., 2002. A landscape perspective of surface-subsurface hydrological exchanges in river corridors. Freshw. Biol. 47, 621-640. https://doi.org/10.1046/j.13652427.2002.00906.x

Mechelke, J., Longrée, P., Singer, H., Hollender, J., 2019. Vacuum-assisted evaporative concentration combined with LC-HRMS/MS for ultra-trace-level screening of organic micropollutants in environmental water samples. Anal. Bioanal. Chem. 411, 2555-2567. https://doi.org/10.1007/s00216-019-01696-3

Moschet, C., Vermeirssen, E.L.M., Singer, H., Stamm, C., Hollender, J., 2015. Evaluation of in-situ calibration of chemcatcher passive samplers for 322 micropollutants in agricultural and urban affected rivers. Water Res. 71, 306-317. https://doi.org/10.1016/j.watres.2014.12.043

Otto, J., Singer, H., Vogler, B., Deuber, F., Longrée, P., Czekalski, N., McArdell, C.S., Götz, C., 2014. Substanzen zur Überprüfung des Reinigungseffekts weitergehender Abwasserbehandlungsverfahren. Technical report on behalf of the Federal Office for the Environment (FOEN).

Posselt, M., Jaeger, A., Schaper, J.L., Radke, M., Benskin, J.P., 2018. Determination of polar organic micropollutants in surface and pore water by high-resolution sampling-direct injection-ultra high performance liquid chromatography-tandem mass spectrometry. Environ. Sci. Process. Impacts 20, 1716-1727. https://doi.org/10.1039/c8em00390d

Radjenović, J., Pérez, S., Petrović, M., Barceló, D., 2008. Identification and structural characterization of 
biodegradation products of atenolol and glibenclamide by liquid chromatography coupled to hybrid quadrupole time-of-flight and quadrupole ion trap mass spectrometry. J. Chromatogr. A 1210, 142-153. https://doi.org/10.1016/j.chroma.2008.09.060

Radke, M., Ulrich, H., Wurm, C., Kunkel, U., 2010. Dynamics and attenuation of acidic pharmaceuticals along a river stretch. Environ. Sci. Technol. 44, 2968-2974. https://doi.org/10.1021/es903091z

Raza, M., Brückner, T., Kübeck, C., Schüth, C., 2018. Pharmaceuticals sorption to soil and biofilm, in: Poster Contribution at 14th Chemistry Conference for Young Scientists by Youth Division of the Royal Flemish Chemical Society. Blankenberge, Belgium.

Reemtsma, T., Berger, U., Arp, H.P.H., Gallard, H., Knepper, T.P., Neumann, M., Quintana, J.B., Voogt, P. De, 2016. Mind the Gap: Persistent and Mobile Organic Compounds - Water Contaminants That Slip Through. Environ. Sci. Technol. 50, 10308-10315. https://doi.org/10.1021/acs.est.6b03338

Rubirola, A., Llorca, M., Rodriguez-Mozaz, S., Casas, N., Rodriguez-Roda, I., Barceló, D., Buttiglieri, G., 2014. Characterization of metoprolol biodegradation and its transformation products generated in activated sludge batch experiments and in full scale WWTPs. Water Res. 63, 21-32. https://doi.org/10.1016/j.watres.2014.05.031

Schaper, J.L., Posselt, M., Bouchez, C., Jaeger, A., Nuetzmann, G., Putschew, A., Singer, G., Lewandowski, J., 2019. Fate of Trace Organic Compounds in the Hyporheic Zone: Influence of Retardation, the Benthic Biolayer, and Organic Carbon. Environ. Sci. Technol. 53, 4224-4234. https://doi.org/10.1021/acs.est.8b06231

Schaper, J.L., Posselt, M., McCallum, J.L., Banks, E.W., Hoehne, A., Meinikmann, K., Shanafield, M.A., Batelaan, O., Lewandowski, J., 2018a. Hyporheic Exchange Controls Fate of Trace Organic Compounds in an Urban Stream. Environ. Sci. Technol. 52, 12285-12294. https://doi.org/10.1021/acs.est.8b03117

Schaper, J.L., Seher, W., Nützmann, G., Putschew, A., Jekel, M., Lewandowski, J., 2018b. The fate of polar trace organic compounds in the hyporheic zone. Water Res. 140, 158-166. https://doi.org/10.1016/j.watres.2018.04.040

Schwarzenbach, R.P., Escher, B.I., Fenner, K., Hofstetter, T.B., Johnson, C.A., Von Gunten, U., Wehrli, B., 2006. The challenge of micropollutants in aquatic systems. Science (80-. ). 313, 1072-1077. https://doi.org/10.1126/science.1127291 
Schwarzenbach, R.P., Gschwend, P.M., Imboden, D.M., 2016. Environmental Organic Chemistry, 3rd ed. Wiley.

Stadlmair, L.F., Grosse, S., Letzel, T., Drewes, J.E., Grassmann, J., 2019. Comprehensive MS-based screening and identification of pharmaceutical transformation products formed during enzymatic conversion. Anal. Bioanal. Chem. 411, 339-351. https://doi.org/10.1007/s00216-018-1442-7

Teasdale, P.R., Batley, G.E., Apte, S.C., Webster, I.T., 1995. Pore water sampling with sediment peepers. Trends Anal. Chem. 14, 250-256. https://doi.org/10.1016/0165-9936(95)91617-2

USEPA, 2013. Pore water sampling. SESCPROC-513-R2. Sci. Ecosyst. Support Div. Athens, GA.

Verleger, H., Schumacher, F., 2012. Auswirkungen von Maßnahmen zur Gewässerentwicklung und zur Verbesserung des Hochwasserschutzes in der Erpe auf den Grundwasserstand. Berlin. Retrieved March 21, 2019, from https://www.berlin.de/senuvk/umwelt/wasser/download/erpe-erlaeuterungsbericht_gw.pdf.

Vermeirssen, E.L.M., Asmin, J., Escher, B.I., Kwon, J.H., Steimen, I., Hollender, J., 2008. The role of hydrodynamics, matrix and sampling duration in passive sampling of polar compounds with Empore ${ }^{\mathrm{TM}}$ SDBRPS disks. J. Environ. Monit. 10,119-128. https://doi.org/10.1039/b710790k

Vermeirssen, E.L.M., Dietschweiler, C., Escher, B.I., Van Der Voet, J., Hollender, J., 2013. Uptake and release kinetics of 22 polar organic chemicals in the Chemcatcher passive sampler. Anal. Bioanal. Chem. 405, 52255236. https://doi.org/10.1007/s00216-013-6878-1

Xie, H., Chen, J., Chen, Q., Chen, C.E.L., Du, J., Tan, F., Zhou, C., 2018a. Development and evaluation of diffusive gradients in thin films technique for measuring antibiotics in seawater. Sci. Total Environ. 618, 1605-1612. https://doi.org/10.1016/j.scitotenv.2017.09.330

Xie, H., Chen, Q., Chen, J., Chen, C.E.L., Du, J., 2018b. Investigation and application of diffusive gradients in thinfilms technique for measuring endocrine disrupting chemicals in seawaters. Chemosphere 200, 351-357. https://doi.org/10.1016/j.chemosphere.2018.02.096

Yongping Zeng, E., Wang, Z., Sojinu, O.S., 2011. Principles and Guidelines of Sampling, Extraction, and Instrumental Analysis Techniques for Measurements of Organic Pollutants in Environmental Matrices. Biophys. Process. Anthropog. Org. Compd. Environ. Syst. 283-314. https://doi.org/10.1002/9780470944479.ch12

Zhang, H., Davison, W., Knight, B., Mcgrath, S., 1998. In situ measurements of solution concentrations and fluxes of trace metals in sells using DGT. Environ. Sci. Technol. 32, 704-710. https://doi.org/10.1021/es9704388 
Zirlewagen, J., Strathmann, M., Schiperski, F., Licha, T., Nödler, K., Hillebrand, O., Idzik, K., 2013. Occurrence and fate of the angiotensin II receptor antagonist transformation product valsartan acid in the water cycle $-\mathrm{A}$ comparative study with selected $\beta$-blockers and the persistent anthropogenic wastewater indicators carbamazepine and acesulfame. Water Res. 47, 6650-6659. https://doi.org/10.1016/j.watres.2013.08.034 